\title{
FLASQUE MODEL STRUCTURES FOR PRESHEAVES
}

\author{
DANIEL C. ISAKSEN
}

\begin{abstract}
By now it is well known that there are two useful (objectwise or local) families of model structures on presheaves: the injective and projective. In fact, there is at least one more: the flasque. For some purposes, both the projective and the injective structure run into technical and annoying (but surmountable) difficulties for different reasons. The flasque model structure, which possesses a combination of the convenient properties of both structures, sometimes avoids these difficulties.
\end{abstract}

\section{INTRODUCTION}

It is well known that there are two useful classes of model structures on simplicial presheaves: the projective and injective. This paper is about a third class of intermediate model structures, called the flasque structures. Flasque presheaves arise on a regular basis in homotopical sheaf theory [BG] [J1] [DI], so it is surprising that these model structures have not been described previously, especially since the proofs (as will be seen) are pretty simple if one takes the right perspective. The goal of this paper is to show that the usefulness of flasque presheaves arises from the fact that there are flasque model structures with convenient properties.

The point of the flasque model structures is that they share some of the advantageous properties of both the projective and injective structures. In the injective model structures, it is very convenient that the cofibrations are easy to describe and every object is cofibrant, but the disadvantage is that the fibrations are not so easy to describe. On the other hand, the projective model structures have the advantage that the fibrations are easy to describe, but the cost is that the cofibrations become more complicated.

The flasque model structures lie somewhere between the projective and injective model structures. By "between", we mean that the weak equivalences are the same, while the class of projective cofibrations is contained in the class of flasque cofibrations and the class of flasque cofibrations is contained in the class of injective cofibrations. Dually, this means that the class of injective fibrations is contained in the class of flasque fibrations and the class of flasque fibrations is contained in the class of projective cofibrations.

One of the confusing aspects of motivic homotopy theory is that there is a wide choice of foundational approaches $[\mathrm{B}] \mathrm{DHI}] \mathrm{Hu}] \mathrm{MV}] \mathrm{M}$, involving presheaves or sheaves, projective or injective cofibrations, sheaves of homotopy groups or localizations with respect to hypercovers, etc. The positive aspect of this circumstance is that in any situation, one can choose the foundational approach that is best suited to the problem at hand.

The flasque model structures are yet another possible foundational choice. As an example of a result that can most easily be proven with the flasque model structures, we show in Theorem 6.8 that motivic stable homotopy groups commute 
with filtered colimits (see also [DI]). In fact, this problem originally motivated this project. The key ingredient in the proof of this theorem is that there are explicit (and verifiable) conditions for an object to be flasque fibrant, although this description is slightly more complicated than for projective fibrant objects. This is entirely unlike the injective model structures, where there is no explicit description of the fibrant objects.

As an another example, we consider Jardine's work on the foundations of stable motivic homotopy theory [J1. Here the projective model structure doesn't suffice for the simple reason that the maps $* \rightarrow \mathbb{P}^{1}$ and $* \rightarrow \mathbb{A}^{1} / \mathbb{A}^{1}-0$ are not projective cofibrations. These are the usual models for the motivic sphere $S^{2,1}$, and it's much more difficult to define spectra with non-cofibrant spheres.

Consequently, the approach in [J1] is to use the injective model structure, where the two models for $S^{2,1}$ are cofibrant. However, many of the proofs in [J1] begin with the observation that injective fibrant presheaves are also flasque. This suggests that the flasque model structure is in fact even more suitable in this context than the injective model structure. Note that the maps $* \rightarrow \mathbb{P}^{1}$ and $* \rightarrow \mathbb{A}^{1} / \mathbb{A}^{1}-0$ are still flasque cofibrations.

In short, the flasque model structures should be added to the collection of frameworks for motivic homotopy theory (along with the projective and injective model structures) because it is just the right tool in some situations.

This paper also answers some questions that arose in [J3 about model structures that are intermediate between the injective and projective. Namely, we show by example that reasonable intermediate model structures do exist, and they are well-behaved in the sense that they are cofibrantly generated (in fact, cellular). Our approach to the local flasque model structure is to perform a left Bousfield localization of the objectwise flasque model structure, rather than to work directly with sheaves of homotopy groups. This localization approach gives the same class of weak equivalences, but it is easier to verify abstract model theoretic properties of the local model structure.

1.1. Related work. We mention a few papers that were absolutely essential in the development of this project. First, we acknowledge [J2 as the seminal paper on the subject of model structures for simplicial presheaves.

Brown and Gersten [BG] defined a flasque model structure for simplicial sheaves rather than presheaves. In a sense, we're doing the presheaf analogue of what they did thirty years ago. In Theorem 4.6] we generalize part of their main result on the existence of certain model structures. Our approach has a significant advantage over $[\mathrm{BG}$. Namely, there is no need for a Noetherian hypothesis on the Grothendieck topology. However, without some kind of such hypothesis, it is not possible to obtain the elegant description of fibrant objects in terms of certain squares being homotopy cartesian. The work of $[\nabla]$ should also be mentioned. This is an axiomatization of the approach of Brown and Gersten. Again, we get the existence of model structures in greater generality but do not obtain the simple description of fibrant objects.

Lárusson $[\mathrm{L}$ has recently used a flasque model structure in the context of complex analysis. His work inspired ours. Actually, his situation is a slight variation on the one that we consider (see Remark 3.4). 
The paper [J3] is about model structures intermediate between the projective and injective. The flasque model structures are a perfect example of the kind of structures studied there.

We should also mention that the definition of flasque presheaf is lifted directly from [J1, which in turn is inspired by BG] .

Finally, we thank Dan Dugger for many helpful observations.

\section{Simplicial PRESheaves}

Recall that a simplicial presheaf on a small site $\mathcal{C}$ is just a contravariant functor from $\mathcal{C}$ to the category $s \mathcal{S} e t$ of simplicial sets. We'll denote the category of presheaves by $s \operatorname{Pre}(\mathrm{C})$.

Many of our results will be stated for arbitrary small sites, but we're really interested in the Nisnevich site of smooth schemes over a fixed ground scheme $S$ because that's the situation that applies to motivic homotopy theory.

The category $s$ Pre $(\mathcal{C})$ is enriched over simplicial sets. This means that for every $F$ in $s$ Pre $(\mathcal{C})$ and every simplicial set $K$, there is a tensor $F \otimes K$ and a cotensor $F^{K}$ with certain adjointness properties. Note that $F \otimes K$ is constructed by taking the objectwise product with $K$, and $F^{K}$ is constructed by taking the objectwise simplicial mapping space out of $K$. The enrichment over simplicial sets also means that there are simplicial mapping spaces $\operatorname{Map}(F, G)$ for every $F$ and $G$ in $s$ Pre $(\mathcal{C})$.

Every object $X$ of $\mathcal{C}$ represents a simplicial presheaf of dimension zero. We will intentionally confuse $X$ with the presheaf that it represents.

If $F$ is any simplicial presheaf in $s \operatorname{Pre}(\mathcal{C})$ and $X$ is any object of $\mathcal{C}$, then $F(X)$ is naturally isomorphic to $\operatorname{Map}(X, F)$. We'll almost always use the latter notation, but it's good to keep in mind that it's just another name for $F(X)$.

We recollect one more construction on simplicial presheaves. For any presheaves $F$ and $G$, there is an internal function object $\underline{\operatorname{Hom}}(F, G)$, which is a simplicial presheaf such that maps $A \rightarrow \underline{\operatorname{Hom}}(F, G)$ correspond bijectively to maps $A \times F \rightarrow$ $G$.

\section{Definition 2.1.}

(a) $A$ map $f$ in sPre(C) is an objectwise weak equivalence if $\operatorname{Map}(X, f)$ is a weak equivalence of simplicial sets for each $X$ in $\mathcal{C}$.

(b) A map $f$ in sPre(C) is an injective cofibration if $\operatorname{Map}(X, f)$ is a cofibration of simplicial sets for each $X$ in $\mathrm{C}$.

(c) A map $f$ in sPre(C) is a projective fibration if $\operatorname{Map}(X, f)$ is a fibration of simplicial sets for each $X$ in $\mathcal{C}$.

The projective cofibrations are defined by a left lifting property with respect to the maps that are both objectwise weak equivalences and projective fibrations (i.e., objectwise acyclic fibrations). Dually, the injective fibrations are defined by a right lifting property with respect to the maps that are both objectwise weak equivalences and injective cofibrations.

The following is a well-known result about homotopy theories of diagram categories (see, for example, $\mathrm{BK}$, [D], and [Jo]).

\section{Theorem 2.2.}

(a) The projective cofibrations, objectwise weak equivalences, and projective fibrations form a simplicial proper cellular model structure on sPre(C). 
(b) The injective cofibrations, objectwise weak equivalences, and injective fibrations form a simplicial proper cellular model structure on sPre $(\mathcal{C})$.

(c) The identity is a left Quillen equivalence from the projective objectwise model structure to the injective objectwise model structure.

The proof of part (a) relies on a standard recognition principle for cofibrantly generated categories Hil, Thm. 11.3.1]. The projective generating cofibrations are maps of the form $X \otimes \partial \Delta^{n} \rightarrow X \otimes \Delta^{n}$, where $n \geq 0$ and $X$ belongs to $\mathcal{C}$. The projective generating acyclic cofibrations are maps of the form $X \otimes \Lambda^{n, k} \rightarrow X \otimes \Delta^{n}$, where $n \geq k \geq 0$ and $X$ belongs to $\mathcal{C}$.

The proof of part (b) is quite a bit harder and relies on some complicated settheoretic arguments. As a result, there is no explicit description of the injective fibrations. This is the chief disadvantage of the objectwise injective model structure.

The proof of part (c) is easy. A projective cofibration is an injective cofibration, and the classes of weak equivalences in the two model categories are identical.

\section{ObJectwise Flasque model Structure}

In this section, we work with an arbitrary small indexing category $\mathcal{C}$. For now, it is not important that $\mathcal{C}$ have a Grothendieck topology. In a few places (such as Theorem 3.7(c) and Corollary 3.15) we need the mild hypothesis that $\mathcal{C}$ contains all finite products. In practice, this is not really a restriction of generality.

Definition 3.1. Let $X$ be any object of $\mathcal{C}$, and let $\mathcal{U}$ be a (possibly empty) finite collection of monomorphisms $U_{i} \rightarrow X$ in $\mathcal{C}$. Define $\cup \mathcal{U}=\cup_{i=1}^{n} U_{i}$ to be the presheaf that is the coequalizer of the diagram

$$
\bigsqcup_{i, j} U_{i} \times_{X} U_{j} \Longrightarrow \coprod_{i} U_{i}
$$

where the top arrow is projection onto the first factor and the bottom arrow is projection onto the second factor.

Beware that $\cup_{i=1}^{n} U_{i}$ is not the presheaf represented by the union (in $\mathcal{C}$ ) of the $U_{i}$ 's. There is no guarantee that this union even exists in $\mathcal{C}$. In fact, in our main application to the category of smooth schemes, these unions generally don't exist: the union of two smooth subschemes of a smooth scheme does not have to be smooth.

Note that there is a canonical map $\cup \mathcal{U} \rightarrow X$, and this map is a monomorphism of presheaves.

When $\mathcal{U}$ is empty, $\cup \mathcal{U}$ is equal to the empty presheaf.

Recall that if $f: F \rightarrow G$ is a map of presheaves and $g: K \rightarrow L$ is a map of simplicial sets, then the pushout product $\boldsymbol{f} \square \boldsymbol{g}$ is the map

$$
G \otimes K \coprod_{F \otimes K} F \otimes L \rightarrow G \otimes L .
$$

A similar definition applies to two maps of simplicial presheaves, except that the tensors are replaced by ordinary products.

\section{Definition 3.2.}

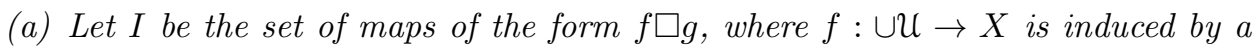
finite collection of monomorphisms into an object $X$ of $\mathcal{C}$ and $g$ is a generating cofibration $\partial \Delta^{n} \rightarrow \Delta^{n}$. 
(b) Let $J$ be the set of maps of the form $f \square g$, where $f: \cup \mathcal{U} \rightarrow X$ is induced by a finite collection of monomorphisms into an object $X$ of $\mathcal{C}$ and $g$ is a generating acyclic cofibration $\Lambda^{n, k} \rightarrow \Delta^{n}$.

We will eventually show that $I$ serves as a set of generating flasque cofibrations, while $J$ serves as a set of generating acyclic flasque cofibrations.

Recall that an $I$-injective is a map having the right lifting property with respect to all elements of $I$, and an $I$-cofibration is a map having the left lifting property with respect to all $I$-injectives (and similarly for $J$-injectives and $J$-cofibrations).

Definition 3.3. A map $f$ in $s P r e(\mathcal{C})$ is a flasque fibration if it is a J-injective.

By the usual adjointness arguments, a map $f: F \rightarrow G$ is flasque if and only if the map

$$
\operatorname{Map}(X, F) \rightarrow \operatorname{Map}(X, G) \times \operatorname{Map}(\cup U, G) \operatorname{Map}(\cup \mathcal{U}, F) .
$$

is a fibration of simplicial sets for all finite collections $\mathcal{U}$ of monomorphisms into an object $X$ of $\mathrm{e}$.

If $f$ is a flasque fibration, then $\operatorname{Map}(X, F) \rightarrow \operatorname{Map}(X, G)$ is a fibration for all $X$ in $\mathcal{C}$. This follows from the existence of the empty collection of monomorphisms into $X$.

A special case of Definition 3.3 tells us that a presheaf $F$ is flasque fibrant if and only if the map $\operatorname{Map}(X, F) \rightarrow \operatorname{Map}(\cup \mathcal{U}, F)$ is a fibration for all finite collections $\mathcal{U}$ of monomorphisms into an object $X$ of $\mathcal{C}$.

Note also that if $Y \rightarrow X$ is a monomorphism in $\mathcal{C}$ and $f: F \rightarrow G$ is a flasque fibration, then the map

$$
\operatorname{Map}(X, F) \rightarrow \operatorname{Map}(X, G) \times \operatorname{Map}(Y, G) \operatorname{Map}(Y, F)
$$

is a fibration. Similarly, if $F$ is flasque fibrant, then $\operatorname{Map}(X, F) \rightarrow \operatorname{Map}(Y, F)$ is a fibration.

Remark 3.4. There are many possible ways to vary the definition of flasque fibrations. The results proved below about flasque model structures would hold just as well for these variations. We let the interested reader check that the arguments below do carry over in the following two situations.

One possible variation is to require that a flasque fibration have the right lifting property with respect to $\cup \mathcal{U} \rightarrow X$ only for collections $\mathcal{U}$ of monomorphisms of size 0 and 1 (or for that matter, for collections up to size $n$ ). Another possible variation is to only consider collections $\mathcal{U}$ such that each monomorphism $U_{i} \rightarrow X$ belongs to some special class of monomorphisms. We have to assume that this special class is closed under base changes, though.

The situation of $[\mathrm{L} \S 12]$ is a combination of the two variations described above, where one considers collections of "Stein inclusions" (a special kind of monomorphism) of size 0 or 1 .

More abstractly, in order to make the inductive proof of Lemma 3.9 work, we need a class $C$ of finite collections of monomorphisms such that $C$ is closed under taking subcollections and is closed under base changes. This last condition means that if $\left\{U_{i} \rightarrow X\right\}$ belongs to $C$ and $Y \rightarrow X$ is any map, then $\left\{U_{i} \times_{X} Y \rightarrow Y\right\}$ also belongs to $C$.

Remark 3.5. The traditional definition of a flasque presheaf $F$ of sets (on the Grothendieck topology of open subsets of a fixed topological space $X$ ) requires 
that each map $F(U) \rightarrow F(V)$ be a surjection of sets for each inclusion $V \rightarrow U$ of open sets of $X$. When $F$ is a discrete simplicial presheaf, our definition of a flasque fibrant presheaf does not exactly correspond to this traditional definition because every map between discrete simplicial sets is a fibration. However, the philosophy is the same because intuitively fibrations can be viewed as a certain kind of surjection, even though this is not technically precise.

Definition 3.6. A map $f$ in $s P r e(\mathcal{C})$ is a flasque cofibration if it has the left lifting property with respect to all objectwise acyclic flasque fibrations.

\section{Theorem 3.7.}

(a) The flasque cofibrations, objectwise weak equivalences, and flasque fibrations form a proper cellular model structure on sPre(C). The set of maps I and $J$ (see Definition [3.2) serve as generating cofibrations and generating acyclic cofibrations.

(b) The identity functor on sPre(C) is a left Quillen equivalence from the objectwise projective model structure to the objectwise flasque model structure and from the objectwise flasque model structure to the objectwise injective model structure.

(c) If $\mathcal{C}$ contains finite products, then the model structure of part (a) is simplicial.

Proof. As for the objectwise projective model structure of Theorem 2.2(a), the proof is an application of a standard recognition principle for cofibrantly generated model categories [Hi Thm. 11.3.1]. In order to apply this principle, we have to check a few things.

First, the category $s \operatorname{Pre}(\mathrm{C})$ contains all small limits and colimits (they're constructed objectwise). Second, the objectwise weak equivalences satisfy the twoout-of-three axiom and the retract axiom. The rest of the hypotheses of $\mathrm{Hi}$, Thm. 11.3.1] are proved below in Lemmas 3.9, 3.10 and 3.11

The simplicial structure of part (c) (of which, as usual, only axiom SM7 is nontrivial) is handled below by Corollary 3.16

For right properness, first note that every flasque fibration is an objectwise fibration. Since pullbacks are constructed objectwise in $s \operatorname{Pre}(\mathcal{C})$, right properness follows from right properness of simplicial sets.

For left properness, we can apply the same argument as in the previous paragraph if we can show that flasque cofibrations are objectwise cofibrations. This is proved below in Lemma 3.8

The rest of this section is dedicated to proving the technical conditions needed in the proof of Theorem 3.7

Lemma 3.8. A projective cofibration is a flasque cofibration, and a flasque cofibration is an injective cofibration. An injective fibration is a flasque fibration, and a flasque fibration is a projective fibration.

Proof. It follows from the definitions that an objectwise acyclic flasque fibration is an objectwise acyclic projective fibration and that a flasque fibration is a projective fibration. Now projective cofibrations are determined by the left lifting property with respect to all objectwise acyclic projective fibrations (and similarly for flasque cofibrations), so a projective cofibration is a flasque cofibration.

If $f: \cup U \rightarrow X$ is a monomorphism and $g: \Lambda^{n, k} \rightarrow \Delta^{n}$ is an acyclic cofibration, then $f \square g$ is an objectwise acyclic injective cofibration. This implies that every 
injective fibration is a flasque fibration, which implies in turn that every flasque cofibration is an injective cofibration.

Lemma 3.9. A map $f: F \rightarrow G$ in sPre(e) is an objectwise acyclic flasque fibration if and only if the map

$$
\operatorname{Map}(X, F) \rightarrow \operatorname{Map}(X, G) \times_{\operatorname{Map}(\cup u, G)} \operatorname{Map}(\cup \mathcal{U}, F)
$$

is an acyclic fibration of simplicial sets for every finite collection $\mathcal{U}$ of monomorphisms into an object $X$ of $\mathcal{C}$. Equivalently, a map is an objectwise acyclic flasque fibration if and only if it is an I-injective.

Proof. The second statement follows from the first by the usual adjointness tricks. To simplify the notation, let $\operatorname{Map}(\mathcal{U}, f)$ be the map under consideration.

First suppose that $\operatorname{Map}(\mathcal{U}, f)$ is an acyclic fibration for every finite collection $\mathcal{U}$. This immediately implies that $f$ is a flasque fibration. Now $\operatorname{Map}(X, F) \rightarrow$ $\operatorname{Map}(X, G)$ is an acyclic fibration for every $X$ in $\mathcal{C}$ (consider the empty collection of monomorphisms into $X$ ). Thus, $f$ is an objectwise weak equivalence.

For the other direction, suppose that $f$ is an objectwise acyclic flasque fibration. From the definitions, this immediately implies that $\operatorname{Map}(\mathcal{U}, f)$ is a flasque fibration. We use induction to show that $\operatorname{Map}(\mathcal{U}, f)$ is an objectwise weak equivalence.

When $n=0$, the presheaf $\cup \mathcal{U}$ is empty. In this case, $\operatorname{Map}(\mathcal{U}, f)$ is just the map $\operatorname{Map}(X, F) \rightarrow \operatorname{Map}(X, G)$, which was assumed to be a weak equivalence.

Now assume that the lemma has been proved for collections of monomorphisms of size at most $n-1$. Let $\mathcal{U}^{\prime}=\left\{U_{1}, \ldots, U_{n-1}\right\}$, and let $\mathcal{U}=\left\{U_{1}, \ldots, U_{n-1}, V\right\}$. First note that $\cup \mathcal{U}$ equals $\cup \mathcal{U}^{\prime} \bigsqcup_{\cup \mathcal{U}^{\prime \prime}} V$, where $\mathcal{U}^{\prime \prime}=\left\{U_{i} \times_{X} V\right\}$ is viewed as a collection of monomorphisms into $V$. Thus $\operatorname{Map}(\cup \mathcal{U}, F)$ equals

$$
\operatorname{Map}\left(\cup \mathcal{U}^{\prime}, F\right) \times_{\operatorname{Map}\left(\cup \mathcal{U}^{\prime \prime}, F\right)} \operatorname{Map}(V, F)
$$

(and similarly for $\operatorname{Map}(\cup \mathcal{U}, G)$ ). Hence we have a diagram

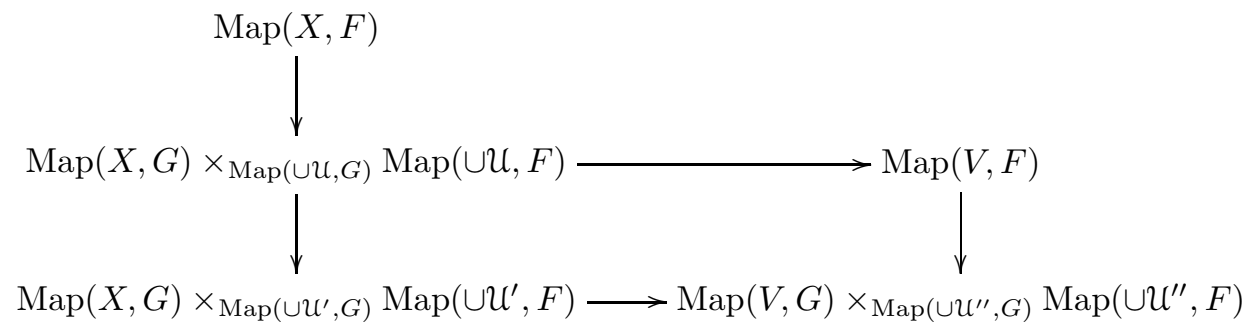

in which the square is a pullback square. The induction assumption tells us that the right vertical arrow is an acyclic fibration, so the lower left vertical arrow is also. The induction assumption also tells us that the composition of the left column is an acyclic fibration, so the two-out-of-three axiom allows us to conclude that $\operatorname{Map}(\mathcal{U}, f)$ is a weak equivalence.

Lemma 3.10. The domains of the maps in I or J (see Definition 3.2) are $\omega$-small.

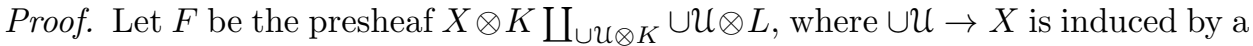
finite collection of monomorphisms into an object $X$ of $\mathcal{C}$ and $K \rightarrow L$ is an inclusion of finite simplicial sets. It suffices to show that each of the three presheaves $X \otimes K$, $\cup \mathcal{U} \otimes K$, and $\cup \mathcal{U} \otimes L$ are $\omega$-small.

Now $-\otimes K$ is left adjoint to the cotensor $(-)^{K}$, and $(-)^{K}$ commutes with filtered colimits because $K$ is finite. Thus it suffices simply to show that $X$ and $\cup \mathcal{U}$ are 
$\omega$-small. Note that $\operatorname{Hom}(X, F)$ is equal to the set of 0 -simplices of the simplicial set $F(X)$; this shows that $X$ is $\omega$-small.

For $\cup \mathcal{U}$, the proof is by induction on the size of $\mathcal{U}$. When $n=0$, the empty presheaf is certainly $\omega$-small.

Now assume that the lemma has been proved for collections of monomorphisms of size at most $n-1$. Let $\mathcal{U}^{\prime}=\left\{U_{1}, \ldots, U_{n-1}\right\}$, and let $\mathcal{U}=\left\{U_{1}, \ldots, U_{n-1}, V\right\}$. First note that $\cup \mathcal{U}$ equals $\cup \mathcal{U}^{\prime} \bigsqcup_{\cup u^{\prime \prime}} V$, where $\mathcal{U}^{\prime \prime}=\left\{U_{i} \times_{X} V\right\}$ is viewed as a collection of monomorphisms into $V$. As above, it suffices to observe that $\cup \mathcal{U}^{\prime}$, $\cup \mathcal{U}^{\prime \prime}$, and $V$ are all $\omega$-small, which follows from the induction assumption.

Lemma 3.11. If a map is a $J$-cofibration, then it is an objectwise acyclic flasque cofibration.

Proof. Suppose that $f$ is a $J$-cofibration. It has the left lifting property with respect to all flasque fibrations by definition. Therefore, $f$ has the left lifting property with respect to all objectwise acyclic flasque fibrations, which makes it a flasque cofibration.

Now we just have to show that a $J$-cofibration is an objectwise weak equivalence. There are two ways of proceeding. One way is to just observe that $J$-cofibrations are objectwise acyclic injective cofibrations because injective fibrations are $J$-injectives by Lemma 3.8. The problem with this approach is that it relies on the existence of the injective model structure, which has a complicated proof. We'll take a more elementary approach.

Recall that a relative $J$-cell complex is a transfinite composition of cobase changes of maps in $J$. Standard arguments imply that every relative $J$-cell complex is a $J$-cofibration. We will first show that relative $J$-cell complexes are objectwise weak equivalences.

By direct inspection a map in $J$ is an objectwise weak equivalence and an objectwise cofibration. Next, a cobase change of a map in $J$ is also an objectwise weak equivalence and an objectwise cofibration. Finally, a transfinite composition of maps that are both objectwise weak equivalences and objectwise cofibrations is again an objectwise weak equivalence and objectwise cofibration.

Now that we know that relative $J$-cell complexes are objectwise weak equivalences, we consider an arbitrary $J$-cofibration $f$. First use the small object argument (with respect to $J$ ) to factor $f$ as $p i$, where $i$ is a relative $J$-cell complex and $p$ is a $J$-injective. Now $f$ lifts with respect to $p$ by assumption, which implies by the retract argument that $f$ is a retract of $i$. Since $i$ is an objectwise weak equivalence by the previous paragraph, so is $f$.

We used in an essential way in the above proof that the map $\cup \mathcal{U} \rightarrow X$ is a monomorphism. Without that, we wouldn't be able to conclude that a cobase change of a map in $J$ is an objectwise weak equivalence. Thus, it's critical that we consider finite collections $\mathcal{U}$ of monomorphisms into an object $X$ of $\mathcal{C}$, not all finite collections of maps into $X$.

It's not surprising that we're forced to consider monomorphisms in $\mathcal{C}$. Otherwise, we would be producing classes of cofibrations that aren't monomorphisms. Although such cofibrations are not axiomatically ruled out, in practice cofibrations are some kind of monomorphism for most useful model categories.

3.12. Internal Function Objects. Our next goal is to show that the objectwise flasque model structure interacts well with respect to internal function objects, in 
the same way that the projective and injective objectwise model structures do. We will also show that the objectwise flasque model structure is simplicial.

Lemma 3.13. If $K \rightarrow L$ is any cofibration of simplicial sets, then

$$
\cup \mathcal{U} \otimes L \coprod_{\cup \mathcal{U} \otimes K} X \otimes K \rightarrow X \otimes L
$$

is a flasque cofibration for any finite collection $\mathcal{U}$ of monomorphisms into an object $X$ of $\mathcal{C}$, and it is objectwise acyclic if $K \rightarrow L$ is a weak equivalence.

Proof. The map $K \rightarrow L$ is a transfinite composition of cobase changes of maps of the form $\partial \Delta^{n} \rightarrow \Delta^{n}$, so the map under consideration is a transfinite composition

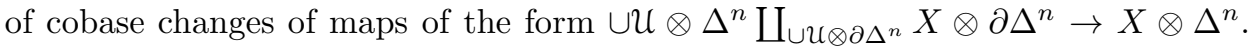
Thus the map is a relative $I$-cell complex, so it is a flasque cofibration.

If $K \rightarrow L$ is a weak equivalence, then it is a retract of a transfinite composition of cobase changes of maps of the form $\Lambda^{n, k} \rightarrow \Delta^{n}$, so the map under consideration is a retract of a transfinite composition of cobase changes of maps of the form $\cup U \otimes \Delta^{n} \bigsqcup_{\cup \mathcal{Q} \otimes \Lambda^{n, k}} X \otimes \Lambda^{n, k} \rightarrow X \otimes \Delta^{n}$. Thus the map is a retract of a relative $J$-cell complex, so it is an objectwise acyclic flasque cofibration.

Recall that we have been working so far with an arbitrary small indexing category $\mathcal{C}$. The next proposition requires the mild hypothesis on $\mathcal{C}$ that it contain finite products. In applications to motivic homotopy theory, this will be no problem.

Proposition 3.14. Let $\mathcal{C}$ be an arbitrary small category that possesses finite products. If $f: F \rightarrow G$ and $g: A \rightarrow B$ are flasque cofibrations, then $f \square g$ is again a flasque cofibration, and it is objectwise acyclic if either $f$ or $g$ is.

Proof. Recall that the flasque cofibrations are the retracts of relative $I$-cell complexes, and the objectwise acyclic flasque cofibrations are the retracts of relative $J$-cell complexes. If $f$ is any map and $g$ is a retract of a transfinite composition of cobase changes of maps in $I$, then $f \square g$ is a retract of a transfinite composition of cobase changes of maps of the form $f \square g^{\prime}$, where $g^{\prime}$ belongs to $I$ (and similarly for $J$ ). Thus it suffices to assume that $g$ belongs to $I$ (for the first part) or to $J$ (for the second part). By the symmetric argument, we can also assume that $f$ belongs to $I$.

Suppose that $f$ is the map $f^{\prime} \square f^{\prime \prime}$, where $f^{\prime}$ is a map $\cup \mathcal{U} \rightarrow X$ and $f^{\prime \prime}$ is the generating cofibration $\partial \Delta^{n} \rightarrow \Delta^{n}$. Similarly, suppose that $g$ is $g^{\prime} \square g^{\prime \prime}$, where $g^{\prime}$ is $\cup \mathcal{V} \rightarrow Y$ and $g^{\prime \prime}$ is $\partial \Delta^{m} \rightarrow \Delta^{m}$. Then careful inspection of the definitions shows that $f \square g$ is isomorphic to $h^{\prime} \square h^{\prime \prime}$, where $h^{\prime}$ is the map $f^{\prime} \square g^{\prime}$ and $h^{\prime \prime}$ is the map $f^{\prime \prime} \square g^{\prime \prime}$. Now $h^{\prime \prime}$ is the map

$$
\Delta^{n} \times \partial \Delta^{m} \coprod_{\partial \Delta^{n} \times \partial \Delta^{m}} \partial \Delta^{n} \times \Delta^{m} \rightarrow \Delta^{n} \times \Delta^{m},
$$

which is a cofibration of simplicial sets, and $h^{\prime}$ is the map $\cup \mathcal{W} \rightarrow X \times Y$, where $\mathcal{W}$ is the collection consisting of maps of the form $U_{i} \times Y \rightarrow X \times Y$ and maps of the form $X \times V_{i} \rightarrow X \times Y$. Thus, $h^{\prime} \square h^{\prime \prime}$ is a flasque cofibration by Lemma 3.13

The same argument works when $g$ belongs to $J$, except that $h^{\prime \prime}$ becomes

$$
\Delta^{n} \times \Lambda^{m, k} \coprod_{\partial \Delta^{n} \times \Lambda^{m, k}} \partial \Delta^{n} \times \Delta^{m} \rightarrow \Delta^{n} \times \Delta^{m},
$$

which is an acyclic cofibration of simplicial sets. 
Note that the above proof (and therefore the following two corollaries) require us to work with arbitrary finite collections of monomorphisms in $\mathcal{C}$, not just collections of size 0 and 1 . This is the essential reason that it is important to work with all finite collections.

Corollary 3.15. Let $\mathcal{C}$ be an arbitrary small category that possesses finite products. If $i: A \rightarrow B$ is a flasque cofibration and $f: F \rightarrow G$ is a flasque fibration, then the map

$$
\underline{\operatorname{Hom}}(B, G) \rightarrow \underline{\operatorname{Hom}}(A, G) \times_{\underline{\operatorname{Hom}}(A, F)} \underline{\operatorname{Hom}}(B, F)
$$

is a flasque fibration, and it is an objectwise weak equivalence if either $i$ or $f$ is.

Proof. This follows immediately by adjointness from Proposition 3.14

The following corollary shows that the flasque model structure of Theorem 3.7 is simplicial.

Corollary 3.16. Let $\mathcal{C}$ be an arbitrary small category that possesses finite products. If $i: A \rightarrow B$ is a flasque cofibration and $f: F \rightarrow G$ is a flasque fibration, then the map

$$
\operatorname{Map}(B, G) \rightarrow \operatorname{Map}(A, G) \times \operatorname{Map}(A, F) \operatorname{Map}(B, F)
$$

is a fibration of simplicial sets, and it is a weak equivalence if either $i$ or $f$ is an objectwise weak equivalence.

Proof. Recall that the space of global sections of the presheaf $\underline{\operatorname{Hom}}(F, G)$ is equal to the simplicial mapping space $\operatorname{Map}(F, G)$. The result now follows from Corollary 3.15

3.17. The projective, flasque, and injective model structures are distinct. We present an elementary example showing that the classes of projective fibrations, flasque fibrations, and injective fibrations are mutually distinct in general.

Let $\mathcal{C}$ be the category with two objects 0 and 1 and two non-identity morphisms from 0 to 1 ; it is indicated by the diagram

$$
0 \Longrightarrow 1 \text {. }
$$

Now a map $F \rightarrow G$ is a projective fibration if and only if the maps $F_{0} \rightarrow G_{0}$ and $F_{1} \rightarrow G_{1}$ are fibrations. It can be checked that $F \rightarrow G$ is a flasque fibration if and only if the maps $F_{0} \rightarrow G_{0}, F_{1} \rightarrow G_{1}$, and both maps $F_{1} \rightarrow F_{0} \times_{G_{0}} G_{1}$ are fibrations. Finally, $F \rightarrow G$ is an injective fibration if and only if the maps $F_{0} \rightarrow G_{0}$ and

are fibrations.

$$
F_{1} \rightarrow\left(F_{0} \times F_{0}\right) \times{ }_{\left(G_{0} \times G_{0}\right)} G_{1}
$$

These three sets of conditions are distinct, as is easily verified by elementary examples. The reader may find it an instructive exercise to show directly that the conditions for injective fibrations implies the conditions for flasque fibrations. This gives a direct verification of Lemma 3.8 in this special case. 


\section{LOCAL MODEL STRUCTURES}

In the previous section, we worked with an index category $\mathcal{C}$ that did not necessarily have a Grothendieck topology. From here on, we must assume that $\mathcal{C}$ does have a Grothendieck topology.

Recall that a hypercover is a certain kind of map of simplicial presheaves SGA4, Exposé V, 7.3] AM DHI. The precise definition is technical and not relevant for us, so we will skip it.

Definition 4.1. The local projective (resp., flasque, injective) model structure on sPre $(\mathrm{C})$ is the left Bousfield localization [Hil Defn. 3.3.1] of the objectwise projective (resp., flasque, injective) model structure at the class of all hypercovers.

This is not the way that the local projective $\mathrm{B}$ and local injective J2 model structures are usually defined. It is more usual (and probably more intuitive) to define local weak equivalences with sheaves of homotopy groups. See [DHI] for a proof that this Bousfield localization approach gives the same class of weak equivalences for the injective and projective model structures. The analogous statement for the flasque model structure appears below in Theorem 4.3

It is formal that these local model structures are left proper, cellular, and simplicial [Hi Thm. 4.1.1]. In fact, these model structures are right proper. One way to see this is to note that the fibrations in any of these model structures are objectwise fibrations. It follows from computing stalks that local weak equivalences are preserved by base change along objectwise fibrations.

Theorem 4.2. The identity functor is a left Quillen equivalence from the local projective model structure to the local flasque model structure and from the local flasque model structure to the local injective model structure.

Proof. In constructing the three left Bousfield localizations, one must choose models for the homotopy colimits of the hypercovers. It doesn't matter which models are chosen; the localizations will be isomorphic.

Since projective cofibrations are flasque cofibrations and flasque cofibrations are injective cofibrations, we can choose models for the homotopy colimits in the projective model structure, and these models will work for the other two structures as well. Thus, all three local model structures are constructed by localizing at the same set of maps.

In order to show that the identity functor gives Quillen equivalences, it suffices because of Theorem 3.7 (b) to apply [Hi Thm. 3.3.20], which tells us that localizations of Quillen equivalent model categories are Quillen equivalent.

The previous theorem does not guarantee that the class of weak equivalences in the local flasque model structure is actually equal to the class of weak equivalences in the local injective or local projective model structure. However, this equality is now not hard to prove.

Theorem 4.3. The local flasque weak equivalences are detected by sheaves of homotopy groups.

Proof. Let $f$ be any local flasque weak equivalence. Factor it into a local acyclic flasque cofibration $i$ followed by a local acyclic flasque fibration $p$. Theorem 4.2 tells us that $i$ is a local acyclic injective cofibration. Thus, $i$ induces isomorphisms 
on sheaves of homotopy groups. On the other hand, $p$ is in fact an objectwise weak equivalence, so it also induces isomorphisms on sheaves of homotopy groups.

Now suppose that $f$ is any map that induces isomorphisms on sheaves of homotopy groups. Factor it into a local acyclic projective cofibration $i$ followed by a local acyclic projective fibration $p$. Theorem 4.2 tells us that $i$ is a local acyclic flasque cofibration. On the other hand, $p$ is an objectwise weak equivalence, so it is certainly a local flasque weak equivalence.

Proposition 4.4. If $i: A \rightarrow B$ is a flasque cofibration and $f: F \rightarrow G$ is a local flasque fibration, then the map

$$
\underline{\operatorname{Hom}}(B, G) \rightarrow \underline{\operatorname{Hom}}(A, G) \times_{\underline{\operatorname{Hom}}(A, F)} \underline{\operatorname{Hom}}(B, F)
$$

is a local flasque fibration, and it is a local weak equivalence if either $i$ or $f$ is.

Proof. This follows by adjointness from a local version of Proposition 3.14. The first part of that result is no problem because the cofibrations in the local flasque model structure are identical to the cofibrations in the objectwise flasque model structure.

For the second part of the local version of Proposition 3.14 note that

$$
B \times F \coprod_{A \times F} A \times G \rightarrow B \times G
$$

is a local weak equivalence for any pair of injective cofibrations $A \rightarrow B$ and $F \rightarrow G$, provided at least one of them is a local weak equivalence. One way to see this is to compute stalks, noting that taking stalks commutes with finite products and pushouts.

4.5. Simplicial sheaves. Recall that the category $s \operatorname{Pre}(\mathcal{C})$ of simplicial presheaves has a full subcategory $s S h(\mathcal{C})$ of simplicial sheaves. The inclusion functor has a left adjoint $a: s \operatorname{Pre}(\mathrm{C}) \rightarrow s S h(\mathrm{C})$, which is called the sheafification functor.

Theorem 4.6. The category $s S h(\mathcal{C})$ has a local flasque model structure in which the weak equivalences are the local weak equivalences, the fibrations are the maps that are flasque fibrations when considered as presheaves, and the cofibrations are generated by the set aI (i.e., the sheafifications of all the maps in I). Sheafification is a left Quillen equivalence from the local flasque model structure on presheaves to the local flasque model structure on sheaves.

Proof. This is an application of a general result about using adjoint functors to translate a model structure from one category to another [Hil Thm. 11.3.2]. That result has several hypothesis, only one of which is not immediately obvious. Namely, we must explain why relative $a J$-cell complexes are local weak equivalences. This follows from the observation that the local weak equivalences are closed under transfinite compositions.

To see that $a$ is a Quillen equivalence, one just needs to use that the natural map $F \rightarrow a F$ is always a local weak equivalence for any simplicial prehseaf $F$ JJ2, Lem. 2.6].

The previous theorem reproves the part of $[\mathrm{BG}]$ that establishes a model structure on the category of sheaves on a Noetherian topological space. At first glance, Theorem 4.6] does not appear to apply because [BG makes no mention of finite 
collections of monomorphisms. However, in the Grothendieck topology of open sets of a fixed topological space, the object $\cup \mathcal{U}$ is always representable.

In fact, Theorem [4.6 is much more general because there is no Noetherian hypothesis on the site. However, we cannot recover the simple identification of fibrant objects in terms of certain homotopy pullback squares. This identification is an important part of $[\mathrm{BG}$ (and its generalization in $[\underline{\mathrm{V}}$ ).

4.7. Nisnevich local model structures. From now on, we specialize to the case of the Nisnevich topology on the category $S m_{S}$ of smooth schemes over a ground scheme $S$ because this is the situation that arises in motivic homotopy theory. In this context, it is not necessary to localize with respect to all hypercovers as in Section 4 Recall that there is a certain class of elementary Nisnevich squares MV. Defn. 3.1.3]

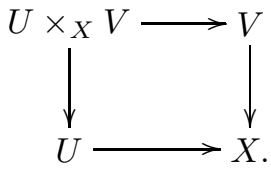

The full definition is not so important, but we will use the fact that $U \rightarrow X$ (and thus also $U \times_{X} V \rightarrow V$ ) is a monomorphism.

The following theorem is proved in [B] Lem. 4.2].

Theorem 4.8. The local projective model structure on $s$ Pre $\left(\mathrm{Sm}_{S}\right)$ is the left Bousfield localization of the objectwise projective model structure at the set of consisting of maps $P \rightarrow X$, where $P$ is the homotopy pushout of

$$
U \longleftarrow U \times_{X} V \longrightarrow V
$$

for all elementary Nisnevich squares.

The homotopy pushouts in the previous theorem are a bit of an annoyance. It would be nicer to have a more concrete description of the maps with respect to which we are localizing. In both the flasque and injective cases, such a description is possible.

Theorem 4.9. The local flasque (resp., injective) model structure on $s \operatorname{Pre}\left(\operatorname{Sm}_{S}\right)$ is the left Bousfield localization of the objectwise flasque (resp., injective) model structure at the set of maps

$$
U \coprod_{U \times_{X} V} V \rightarrow X
$$

for all elementary Nisnevich squares.

Proof. Recall that localizations of Quillen equivalent model categories are Quillen equivalent [Hi, Thm. 3.3.20]. Therefore, if we localize the flasque or injective objectwise model structure at the set of maps $P \rightarrow X$ (as in the previous theorem), then Theorems 3.7 (b) and 4.8 tell us that we get the desired local model structure. But in the flasque and injective model structures, $U \times_{X} V \rightarrow V$ is a cofibration, so the ordinary pushout $U \coprod_{U \times_{X} V} V$ is a model for the homotopy pushout.

The above theorem demonstrates one of the ways in which the flasque (or injective) model structure is more convenient than the projective model structure. The localization is slightly more concrete - there aren't any homotopy pushouts involved. 
Theorem 4.8 formally leads to the following characterization [B, Lem. 4.1]. An object $F$ of $s \operatorname{Pre}\left(\mathrm{Sm}_{S}\right)$ is local projective fibrant if and only if:

(1) $F$ is projective fibrant;

(2)

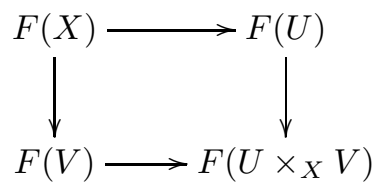

is homotopy cartesian for all elementary Nisnevich squares.

Because of Theorem 4.9 we can improve on this characterization for the flasque and injective model structures.

Corollary 4.10. An object $F$ of $s$ Pre $\left(S m_{S}\right)$ is local flasque (resp., injective) fibrant if and only if:

(1) $F$ is flasque (resp., injective) fibrant;

(2) the map

$$
F(X) \rightarrow F(U) \times_{F\left(U \times_{X} V\right)} F(V)
$$

is an acyclic fibration for all elementary Nisnevich squares.

Proof. This follows from formal properties of left Bousfield localizations Hi Thm. 4.1.1(2)] (see DHI, Cor. 7.1] for a similar argument).

The previous result shows exactly why the flasque model structure is a good compromise between the projective and injective model structures. Condition (1) for the injective model structure is very complicated. However, condition (1) for the flasque structure is much simpler. Condition (2) doesn't work for the projective structure because a messier homotopy pullback statement is required.

\section{Motivic Model Structures}

Definition 5.1. The motivic projective (resp., flasque, injective) model structure on sPre $\left(\mathrm{Sm}_{S}\right)$ is the left Bousfield localization of the local projective (resp., flasque, injective) model structure at the set of maps $X \rightarrow X \times \mathbb{A}^{1}$.

The maps $X \rightarrow X \times \mathbb{A}^{1}$ are induced by the inclusion $0 \rightarrow \mathbb{A}^{1}$.

It is formal that these motivic model structures are left proper, cellular, and simplicial Hi Thm. 4.1.1]. In fact, they are also right proper, but this requires some extra work [MV] 2.2.7] (beware that this reference reverses the definition of left proper and right proper).

Similarly to the local projective model structure, an object $F$ is motivic projective fibrant if and only if:

(1) $F$ is projective fibrant;

(2)

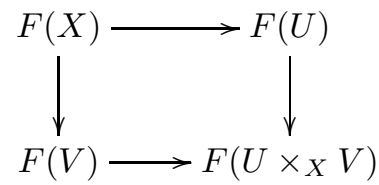

is homotopy cartesian for all elementary Nisnevich squares;

(3) $F\left(X \times \mathbb{A}^{1}\right) \rightarrow F(X)$ is a weak equivalence for all $X$ in $\mathcal{C}$. 
As in Corollary 4.10, we can improve on this characterization for the flasque and injective model structures.

Corollary 5.2. An object $F$ is motivic flasque (resp., injective) fibrant if and only if:

(1) F is flasque (resp., injective) fibrant;

(2) the map is an acyclic fibration;

$$
F(X) \rightarrow F(U) \times_{F\left(U \times_{X} V\right)} F(V)
$$

(3) $F\left(X \times \mathbb{A}^{1}\right) \rightarrow F(X)$ is an acyclic fibration for all $X$ in $\mathcal{C}$.

Proof. As in the proof of Corollary 4.10 this follows from [Hil Thm. 4.1.1(2)].

We present one consequence of Corollary[5.2 which will be important later. Note that the injective analogue of this statement is false.

Proposition 5.3. The class of motivic flasque fibrant objects is closed under filtered colimits.

Proof. Condition (1) of Corollary 5.2 is preserved by filtered colimits because the generating objectwise acyclic flasque cofibrations (i.e., the set $J$ of Definition 3.2) have compact domains. Conditions (2) and (3) are straightforward (see also [J1, $\S 1.3])$.

Proposition 5.4. If $A$ is flasque fibrant and $F$ is motivic flasque fibrant, then $\underline{\operatorname{Hom}}(A, F)$ is also motivic flasque fibrant.

Proof. From Proposition 4.4 we know that $\underline{\operatorname{Hom}}(A, F)$ is local flasque fibrant. By [Hi. Thm. 4.1.1(2)], we only need to show that the map $\underline{\operatorname{Hom}}(A, F) \rightarrow *$ has the right lifting property with respect to every map of the form

$$
X \otimes \Delta^{n} \coprod_{X \otimes \Lambda^{n, k}}\left(X \times \mathbb{A}^{1}\right) \otimes \Lambda^{n, k} \rightarrow\left(X \times \mathbb{A}^{1}\right) \otimes \Delta^{n} .
$$

Now this map is an objectwise acyclic flasque cofibration, so the lifting property is satisfied.

In analogy to Corollary 3.15 and Proposition 4.4 it is natural to consider the map

$$
\underline{\operatorname{Hom}}(B, G) \rightarrow \underline{\operatorname{Hom}}(A, G) \times \underline{\operatorname{Hom}(A, F)} \underline{\operatorname{Hom}}(B, F)
$$

for $i: A \rightarrow B$ a flasque cofibration and $f: F \rightarrow G$ a motivic flasque fibration. However, we do not yet know how to show that this map is a motivic flasque fibration.

\section{Stable motivic Model Structures}

6.1. Pointed model structures. In stable motivic homotopy theory, we're actually interested in the pointed version of what we've been discussing so far, i.e., the category $\operatorname{sPre}\left(S m_{S}\right)_{*}$ of presheaves of pointed simplicial sets. Of course, this is just an undercategory of $s \operatorname{Pre}\left(S m_{S}\right)$, so we obtain formally the pointed objectwise flasque model structure, the pointed local flasque model structure, and the pointed motivic flasque model structure [Hi Thm. 7.6.5].

These pointed model structures are still simplicial model categories with the obvious pointed analogue of tensor. Namely, if $F$ is any pointed presheaf and $K$ is 
any simplicial set, then $F \otimes K$ is the pointed presheaf defined by $(F \otimes K)(U)=$ $F(U) \wedge K_{+}$.

Recall that the fibrant objects in the pointed categories are simply the pointed presheaves that are fibrant in the corresponding unpointed category. On the other hand, the cofibrant objects are different; instead of studying $\phi \rightarrow A$, we now look at $* \rightarrow A$. We're going to need to know that some of the basic objects of motivic homotopy theory are flasque cofibrant pointed presheaves.

Lemma 6.2. If $X \rightarrow Y$ is any monomorphism in $S m_{S}$, then $Y / X$ is a flasque cofibrant pointed presheaf.

Proof. Just consider the pushout square

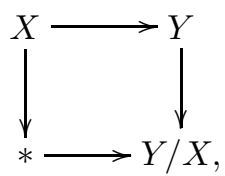

in which the top arrow is a flasque cofibration. Therefore, the bottom arrow is also a flasque cofibration.

The objects mentioned in the following lemma are central to the construction of stable motivic homotopy theory.

Lemma 6.3. In $\operatorname{sPre}\left(S m_{S}\right)_{*}$, the following pointed presheaves are flasque cofibrant:

(1) The presheaf $\mathbb{P}^{1}$, pointed at $\infty$ (or at any other rational point).

(2) The presheaf $\mathbb{A}^{1}-0$, pointed at 1 (or at any other rational point).

(3) The presheaf $\mathbb{A}^{1} / \mathbb{A}^{1}-0$.

(4) The presheaf $\Sigma\left(\mathbb{A}^{1}-0\right)$.

Proof. The first three examples are special cases of the previous lemma. For the last one, we just need to show that the map

$$
* \otimes S^{1} \coprod_{* \otimes *}\left(\mathbb{A}^{1}-0\right) \times * \rightarrow\left(\mathbb{A}^{1}-0\right) \otimes S^{1}
$$

is a flasque cofibration (by the definition of suspension). This is exactly what Lemma 3.13 says.

The category $s \operatorname{Pre}\left(\mathrm{Sm}_{S}\right)_{*}$ still has internal function objects, which we denote by $\underline{\mathrm{Hom}}_{*}(F, G)$. However, we can't prove the pointed analogues of Proposition 3.14 and Corollary 3.15 because that would require taking smash products of representable functors instead of products of representable functors. Nevertheless, we have the following useful result.

Proposition 6.4. If $A$ is a flasque cofibrant pointed presheaf and $F$ is a flasque (resp., local flasque, motivic flasque) fibrant pointed presheaf, then $\underline{\operatorname{Hom}}_{*}(A, F)$ is also a flasque (resp., local flasque, motivic flasque) fibrant pointed presheaf.

Proof. Consider the pullback square

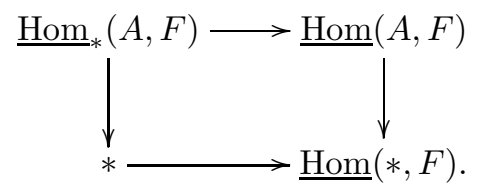


We are interested in the left vertical arrow, which is a base change of the right vertical arrow. Thus, we just have to show that the right vertical arrow is a flasque (resp., local flasque, motivic flasque) fibration. For the first two cases, this follows from Corollary 3.15 and Proposition 4.4.

The third (motivic) case requires only slightly more work. First note that both objects $\underline{\operatorname{Hom}}(A, F)$ and $\underline{\operatorname{Hom}}(*, F)$ are motivic flasque fibrant by Proposition 5.4. We already know that the map between them is a flasque fibration from the previous paragraph. From [Hil Prop. 3.3.16(1)] (a general result describing fibrations between fibrant objects in a localization), we conclude that the map is in fact a motivic flasque fibration.

Recall that for any pointed presheaf $F$, the presheaf $\Omega^{2,1} F$ is defined to be $\underline{\operatorname{Hom}}_{*}(T, F)$, where $T$ is some chosen model for the motivic sphere $S^{2,1}$. For different purposes, authors have chosen $T$ to be $\mathbb{P}^{1}, \mathbb{A}^{1} / \mathbb{A}^{1}-0$, or $\Sigma\left(\mathbb{A}^{1}-0\right)$. In the following corollary, it doesn't matter which of these three are used.

Corollary 6.5. If $F$ is a motivic flasque fibrant pointed presheaf, then $\Omega^{2,1} F$ is also a motivic flasque fibrant pointed presheaf.

Proof. This is a straightforward combination of Lemma 6.3 with Proposition 6.4

The above corollary is a great example of the advantage of the flasque model structures over the projective model structures. Since $* \rightarrow \mathbb{P}^{1}$ and $* \rightarrow \mathbb{A}^{1} /\left(\mathbb{A}^{1}-0\right)$ are not projective cofibrations, one has to choose carefully a model for $S^{2,1}$ when working with the projective structures. With the flasque (or injective) structures, there is no such problem.

6.6. Motivic spectra. One of the key applications of the motivic flasque model structure is for stable motivic homotopy theory. Normally people use the injective structure because they need $* \rightarrow \mathbb{P}^{1}$ (or $\left.* \rightarrow \mathbb{A}^{1} / \mathbb{A}^{1}-0\right)$ to be a cofibration. Of course, this map is also a flasque cofibration, so the general machinery for producing stable model structures $\mathrm{Ho}$ works fine.

To illustrate this point, we will describe a theorem that can be proved only with the flasque model structure (as far as we know). The proof of the theorem will require certain properties that neither the projective nor injective model structures possess.

We briefly review the construction of naive Bousfield-Friedlander motivic spectra. All of what follows would work just as well for motivic symmetric spectra, but we use naive motivic spectra because they are easier to describe and they suffice for our specific purpose.

A naive motivic spectrum $E$ is a sequence $\left\{E_{n}\right\}$ of pointed simplicial presheaves together with structure maps $E_{n} \rightarrow \Omega^{2,1} E_{n+1}$. The definition of maps of naive motivic spectra is obvious.

In order to construct the stable motivic model structure, we can start with the projective, flasque, or injective model structure on pointed simplicial presheaves. We'll start with the flasque model structure.

We only need a few facts about the stable motivic model structure on naive motivic spectra. First, a spectrum $E$ is fibrant if and only if each $E_{n}$ is flasque fibrant and the maps $E_{n} \rightarrow \Omega^{2,1} E_{n+1}$ are (unstable) motivic weak equivalences. Second, a map $E \rightarrow F$ of fibrant spectra is a stable motivic weak equivalence if and 
only if each $E_{n} \rightarrow F_{n}$ is a motivic weak equivalence of pointed simplicial presheaves. Third, the standard models for the sphere spectra $S^{p, q}$ are cofibrant spectra.

Proposition 6.7. Fibrant motivic spectra are closed under filtered colimits.

Proof. Let $\left\{E^{i}\right\}$ be filtered system of naive motivic spectra, and let $E$ be $\operatorname{colim}_{i} E^{i}$. Then $E_{n}$ equals $\operatorname{colim}_{i} E_{n}^{i}$, so it is a motivic flasque fibrant pointed simplicial presheaf by Proposition 5.3

By Corollary [6.5 $\Omega^{2,1} E_{n+1}^{i}$ is a motivic flasque fibrant pointed simplicial presheaf, so the structure map $E_{n}^{i} \rightarrow \Omega^{2,1} E_{n+1}^{i}$ is a motivic weak equivalence between motivic flasque fibrant pointed simplicial presheaves. Therefore, it is in fact an objectwise weak equivalence (this is a general property of localizations). It follows that $\operatorname{colim}_{i} E_{n}^{i} \rightarrow \operatorname{colim}_{i} \Omega^{2,1} E_{n+1}^{i}$ is also an objectwise weak equivlence and hence a motivic weak equivalence. This last map is just the structure map $E_{n} \rightarrow \Omega^{2,1} E_{n+1}$ because $\Omega^{2,1}(-)$ commutes with filtered colimits.

Theorem 6.8. Let $\left\{E^{i}\right\}$ be a filtered system of motivic spectra. The natural map $\operatorname{colim}_{i} \pi_{p, q} E^{i} \rightarrow \pi_{p, q}\left(\operatorname{hocolim}_{i} E^{i}\right)$ is an isomorphism for all $p$ and $q$ in $\mathbb{Z}$.

Proof. We may assume that each $E^{i}$ is a fibrant motivic spectrum. By Proposition 6.7 so is $\operatorname{colim}_{i} E^{i}$. Consider the map

$$
\underset{i}{\operatorname{colim}} \pi_{0} \operatorname{Map}\left(S^{p, q}, E^{i}\right) \rightarrow \pi_{0} \operatorname{Map}\left(S^{p, q}, \operatorname{colim}_{i} E^{i}\right) .
$$

This is an isomorphism because $\operatorname{Map}\left(S^{p, q},-\right)$ commutes with filtered colimits.

It only remains to show that the natural map $\operatorname{hocolim}_{i} E^{i} \rightarrow \operatorname{colim}_{i} E^{i}$ is a stable motivic weak equivalence. It suffices to show that if $\left\{E^{i}\right\}$ and $\left\{F^{i}\right\}$ are filtered systems of motivic fibrant spectra and each $E^{i} \rightarrow F^{i}$ is a stable motivic weak equivalence, then $\operatorname{colim}_{i} E^{i} \rightarrow \operatorname{colim}_{i} F^{i}$ is a stable motivic weak equivalence.

Since each $E^{i} \rightarrow F^{i}$ is a stable motivic weak equivalence between fibrant spectra, each $E_{n}^{i} \rightarrow F_{n}^{i}$ is a motivic weak equivalence between motivic flasque fibrant pointed simplicial presheaves. As observed in the previous proof, each map $E_{n}^{i} \rightarrow F_{n}^{i}$ is in fact an objectwise weak equivalence. It follows that $\operatorname{colim}_{i} E_{n}^{i} \rightarrow \operatorname{colim}_{i} F_{n}^{i}$ is still an objectwise weak equivalence, and this implies that $\operatorname{colim} E_{i} \rightarrow \operatorname{colim} F_{i}$ is a stable motivic weak equivalence.

\section{REFERENCES}

[SGA4] M. Artin, A. Grothendieck, and J. L. Verdier, Théorie des Topos et Cohomologie Étale des Schemas, Lecture Notes in Math., Vol. 270, Springer, 1972.

[AM] M. Artin and B. Mazur, Étale Homotopy, Lecture Notes in Math., Vol. 100, Springer, 1969.

[B] B. A. Blander, Local projective model structures on simplicial presheaves, $K$-Theory 24 (2001), no. 3, 283-301.

[BG] K. S. Brown and S. M. Gersten, Algebraic K-theory as generalized sheaf cohomology, in Algebraic K-theory, I: Higher K-theories (Proc. Conf., Battelle Memorial Inst., Seattle, 1972), 266-292, Lecture Notes in Math., Vol. 341, Springer, 1973.

[BK] A. K. Bousfield and D. M. Kan, Homotopy limits, completions and localizations, Lecture Notes in Math., Vol. 304, Springer, 1972.

[D] D. Dugger, Universal homotopy theories, Adv. in Math. 164 (2001), 144-176.

[DHI D. Dugger, S. Hollander, and D. C. Isaksen, Hypercovers and simplicial presheaves, Math. Proc. Cambridge Philos. Soc., to appear.

[DI] D. Dugger and D. C. Isaksen, Motivic cell structures, preprint.

[Hi] P. S. Hirschhorn, Model Categories and Their Localizations, Mathematical Surveys and Monographs, Vol. 99, Amer. Math. Soc., 2003. 
[Ho] M. Hovey, Spectra and symmetric spectra in general model categories, J. Pure Appl. Algebra 165 (2001), no. 1, 63-127.

[Hu] P. Hu, S-modules in the category of schemes, Mem. Amer. Math. Soc. 161 (2003), no. 767.

[J1] J. F. Jardine, Motivic symmetric spectra, Doc. Math. 5 (2000), 445-552.

[J2] J. F. Jardine, Simplicial presheaves, J. Pure Appl. Algebra 47 (1987), no. 1, 35-87.

[J3] J. F. Jardine, Intermediate model structures for simplicial presheaves, preprint.

[Jo] A. Joyal, Letter to A. Grothendieck, 1984.

[L] F. Lárusson, Model structures and the Oka principle, preprint.

[M] F. Morel, Théorie homotopique des schémas, Astérisque, Vol. 256, 1999.

[MV] F. Morel and V. Voevodsky, $\mathbb{A}^{1}$-homotopy theory, Inst. Hautes Etudes Sci. Publ. Math. 90 (2001), 45-143.

[V] V. Voevodsky, Homotopy theory of simplicial sheaves in completely decomposable topologies, preprint.

Department of Mathematics, Wayne State University, Detroit, Mi 48202

E-mail address: isaksen@math.wayne.edu 